\title{
Phase locking in quantum and classical oscillators: polariton condensates, lasers, and arrays of Josephson junctions
}

\author{
P. R. Eastham, M. H. Szymanska and P. B. Littlewood \\ Cavendish Laboratory, Madingley Road, Cambridge, CB3 OHE. United Kingdom.
}

(Dated: November 1, 2018)

\begin{abstract}
We connect three phenomena in which a coherent electromagnetic field could be generated: polariton condensation, phase-locking in arrays of underdamped Josephson junctions, and lasing. All these phenomena have been described using Dicke-type models of spins coupled to a single photon mode. These descriptions may be distinguished by whether the spins are quantum or classical, and whether they are strongly or weakly damped.
\end{abstract}

\section{INTRODUCTION}

Phase-locking 1] of coupled oscillators is a well-known phenomenon in nonlinear dynamics. The generation of coherent radiation from Josephson junction arrays 2] is but one example for macroscopic oscillators. But phaselocking exists not only in classical systems but in quantum models. Here there are two basic paradigms for coherence of microscopic oscillators: Bose-Einstein condensation (BEC), which is responsible for superfluidity and superconductivity, and lasing.

Although BEC, lasing, and classical phase-locking all involve collective coherent behaviour, they are usually described in very different terms. With some exceptions [3], descriptions of BEC and lasing are given in quantummechanical language, which gives the impression that these phenomena derive from quantum mechanics. Descriptions of Bose condensation sometimes go further, suggesting that the condensate itself is a quantum mechanical object. Nevertheless, it is unclear how these phenomena differ from classical phase-locking.

The aim of this paper is to clarify the relationships amongst BEC, lasing, and classical phase-locking, and hence the extent to which one can describe the coherence in Bose condensates and lasers as "quantum" or "classical". To do this, we will consider Bose condensation of cavity polaritons, phase-locking in arrays of coupled Josephson junctions, and lasing. The simplest theoretical models of these phenomena are in fact similar, and admit some controlled solutions, enabling us to compare them cleanly.

\section{CAVITY POLARITON CONDENSATION}

A cavity polariton [4, 5] is the quantum of the electromagnetic field in an optical cavity containing a dielectric. It is the confined version of the bulk polariton considered many years ago by Hopfield 6], which is formed from propagating photons coupled to electronic excitations such as excitons. Since polaritons are photons coupled to other excitations they are bosons, and therefore might be candidates for Bose condensation.

While the idea of a Bose condensate of bulk polaritons has been discussed for many years 7, 8], it would be an unusual type of condensate. This is because lowenergy polaritons are merely long-wavelength photons, which are not conserved particles. Thus the polaritons cannot condense in the ground state, making the bulk polariton condensate an intrinsically non-equilibrium phenomenon. However, a condensate of cavity polaritons is not necessarily a non-equilibrium phenomenon, because the lifetime of the low-energy cavity polaritons is finite. If this lifetime were long compared with the thermalisation time, one could consider the thermal equilibrium of a population of polaritons, treated as conserved particles. This is the normal situation for Bose condensation.

Eastham and Littlewood 9, 10, 11] have considered such quasi-equilibrium polariton condensation in a toy model. The model is appropriate to localised electronic excitations, such as excitons bound to impurities or localised on quantum dots, dipole coupled to a single mode of a three-dimensional cavity. It has the Hamiltonian

$$
H=\omega_{c} \psi^{\dagger} \psi+\sum_{i}\left[E_{i} S_{i}^{z}+\frac{g}{\sqrt{N}}\left(S_{i}^{+} \psi+\psi^{\dagger} S_{i}^{-}\right)\right] .
$$

$\psi^{\dagger}$ is the creation operator for a cavity photon, with energy $\omega_{c}$. The dielectric is modelled as a set of $N$ two-level systems, with the $i^{\text {th }}$ two-level system described by the spin-half operators $\vec{S}_{i}$. The eigenstates of $S_{i}^{z}$ correspond to the presence or absence of an excitation on site $i$.

The model (1) is the Dicke model 12 of quantum optics, which has been studied in many different regimes. To apply it to polariton condensation one should find its ground state or partition function, fixing the total number of excitations

$$
N_{e x}=\psi^{\dagger} \psi+\sum_{i}\left(S_{i}^{z}+\frac{1}{2}\right)
$$

which is conserved by the Hamiltonian (11). The constraint on $N_{\text {ex }}$ can be dealt with by introducing a chemical potential $\mu$, so that one works with the unconstrained effective Hamiltonian $H_{\text {eff }}=H-\mu N_{e x} . H_{\text {eff }}$ is the same as $H$, except that the photon and exciton energies $\omega_{c}$ and $E_{i}$ are shifted by the chemical potential.

One can write down the ground-state wavefunction of $H_{\text {eff }}$ by generalising the standard wavefunction for a Bose condensate. For bosons with creation operator $b^{\dagger}$, the 
ground-state is the coherent state

$$
e^{\lambda b^{\dagger}}|\mathrm{vac}\rangle
$$

In general, polaritons are superpositions of an excitation of the cavity mode and an excitation of the dielectric. Thus the generalisation of (2) to describe polariton condensation is

$$
\exp \left(\lambda \psi^{\dagger}+\sum_{i} e^{i \phi_{i}} w_{i} S_{i}^{+}\right)|\mathrm{vac}\rangle
$$

where $\lambda, w_{i}$ and $\phi_{i}$ are variational parameters. Minimizing $\left\langle H_{\text {eff }}\right\rangle$ over these parameters gives an equation for $\lambda$ which is analogous to the BCS gap equation:

$$
\left(\omega_{c}-\mu\right) \lambda=\frac{g^{2} \lambda}{N} \sum_{i} \frac{1}{\sqrt{\left(E_{i}-\mu\right)^{2}+4 g^{2}|\lambda|^{2}}} .
$$

The polariton condensate (3) is a superposition of coherent states of the dielectric and the electromagnetic field. It has a finite expectation value for the amplitude of the cavity field, $\langle\psi\rangle$, and the electronic polarisation $\left\langle S_{i}^{-}\right\rangle$. The $\phi_{i}$ are the phase differences between the electronic polarisation and the cavity field. They are fixed by the dipole interaction term in (1), which is responsible for the phase locking: it ensures that all the oscillators with a finite polarisation are mutually coherent, $\phi_{i}=\phi$, when the energy is minimised.

To make the connection to phase-locking more explicit, we note that the gap equation is a special case of the condition for the dynamics of the spins to synchronize at frequency $\mu$. In a frame rotating at this frequency, the Heisenberg equations of motion corresponding to the Hamiltonian (10) are

$$
\begin{aligned}
i \dot{\psi} & =\left(\omega_{c}-\mu\right) \psi+\frac{g}{\sqrt{N}} \sum_{i} S_{i}^{-} \\
i \dot{S}_{i}^{-} & =\left(E_{i}-\mu\right) S_{i}^{-}-\frac{2 g}{\sqrt{N}} S_{i}^{z} \psi \\
i \dot{S}_{i}^{z} & =\frac{g}{\sqrt{N}}\left(S_{i}^{+} \psi-\psi^{\dagger} S_{i}^{-}\right)
\end{aligned}
$$

In an unsynchronized state, the sum on the right-hand side of Eq. 5 is of order $\sqrt{N}$, so $\psi$ is of order 1. For such a $\psi$ the spins are free to leading order in $N$ due to the scaling of the coupling constant. The spin on site $i$ simply precesses around the $\mathrm{z}$ axis at its natural frequency $\left(E_{i}-\mu\right)$. In a synchronized state, part of the sum in Eq. [5 will be of order $N$, so $\psi$ will be of order $\sqrt{N}$. Such a field gives a finite contribution to the effective magnetic field on each spin. The dynamics of $\left\langle S_{i}^{-}\right\rangle$in this effective field contains a static component. Substituting this static component into (5) and setting $\dot{\psi}=0$ gives a self-consistency condition on the synchronized states,

$$
\left(\omega_{c}-\mu\right) \lambda=\frac{g^{2} \lambda}{N} \sum_{i} \frac{2\left\langle S_{i}^{z \prime}\right\rangle_{0}}{\sqrt{\left(E_{i}-\mu\right)^{2}+4 g^{2}|\lambda|^{2}}} .
$$

This condition is a generalisation of (4), in which the unit numerator in the $i^{\text {th }}$ term of the sum becomes $2\left\langle S_{i}^{z \prime}\right\rangle_{0}$. $S_{i}^{z \prime}$ is the component of spin $i$ along its effective field, and \langle\rangle$_{0}$ denotes an expectation value in the initial state. The particular choice of "initial conditions" that appears in Eq. 4 corresponds to a thermal (here $\mathrm{T}=0$ ) occupation of the quasiparticle states, producing a solution of Eq. 1 of the lowest free energy.

The self-consistent approach suggests that a mean-field theory in the amplitude of the cavity mode is exact as $N \rightarrow \infty$. This can be formally demonstrated by constructing the partition function as a path integral, which can be evaluated using saddle-point techniques. Physically, the mean-field theory is exact because the cavity mode is coupled to many electronic states, and so should have relatively small fluctuations. A consequence is that condensation in the model (10) is, in the limit of a large system, no more than the phase-locking of classical coupled oscillators.

\section{JOSEPHSON JUNCTION ARRAYS}

A different system which can be described by models similar to (11) is a Josephson junction array in a microwave cavity. Phase-locking in that system was considered in 1970 by Tilley 13], in a model describing junctions connected in series in a single-mode cavity. He considered the fully synchronized states of the array, in which each junction oscillates at the same frequency. While there are many such states, differing in locking frequency, phase configuration and photon number, he showed that one particular state is selected by the driving current and cavity losses.

To compare locking in Josephson junctions to polariton condensation, we use the angular momentum representation 14] of a Josephson junction. Each junction is represented by a spin $S$, whose magnitude is half the total number of condensed Cooper pairs in the two superconductors connected by the junction. $S^{z}$ is half the difference between the number of condensed pairs on either side of the junction, while $S^{+}$and $S^{-}$transfer condensed pairs across the junction. These operators obey the usual angular momentum commutation rules to the extent that Cooper pairs can be treated as structureless bosons, which should be a good approximation in the weak-coupling limit $\Delta \ll \omega_{D}$. Note that $S$ is usually much larger than 1 - the junctions are themselves macroscopic objects. 
microwave resonance is 15,16$] 32$

$$
H=\omega_{c} \psi^{\dagger} \psi+\sum_{i}\left[K_{i}\left(S_{i}^{z}\right)^{2}+\frac{g_{i}}{\sqrt{N}}\left(S_{i}^{+}-S_{i}^{-}\right)\left(\psi-\psi^{\dagger}\right)+j_{i}\left(S_{i}^{+}+S_{i}^{-}\right)+\nu_{i} S_{i}^{z}\right] .
$$

$j_{i}$ corresponds to the standard Josephson tunnelling, $g_{i}$ to the photon assisted tunnelling, $K_{i}$ to the charging energy of the junction, and $\nu_{i}$ to a voltage bias across the junction. To bring (9) as close as possible to the polariton condensation problem, we neglect the standard Josephson couplings $j_{i}$ and make the rotating-wave approximation. This gives the Hamiltonian

$$
H_{\mathrm{JJ}} \approx \omega_{c} \psi^{\dagger} \psi+\sum_{i}\left[K_{i}\left(S_{i}^{z}\right)^{2}+\frac{g_{i}}{\sqrt{N}}\left(S_{i}^{+} \psi+\psi^{\dagger} S_{i}^{-}\right)+\nu_{i} S_{i}^{z}\right] .
$$

Since (10) conserves $N_{\text {ex }}$, we may consider its behaviour at fixed $N_{\text {ex }}$ 15. This problem should be exactly solvable in the limit $N \rightarrow \infty$ using a mean-field theory in the amplitude of the cavity mode.

To construct the mean-field theory for $H_{\mathrm{JJ}}^{\prime}=H_{\mathrm{JJ}}-\mu N_{\mathrm{ex}}$, we should follow the same self-consistent approach that was used for polariton condensation. This is complicated, however, by the presence of the charging energy. Because of this term, the single-spin effective Hamiltonian of the mean-field theory is nonlinear. Instead of directly tackling this problem, we will follow the variational procedure used for polariton condensation. While this corresponds exactly to the mean-field theory for the polariton condensate, it will only be an approximation to the mean-field theory of the array. This is because (3) is the ground state of the effective Hamiltonian which occurs in the mean-field theory of polariton condensation, but not of that which occurs for the array.

Looking for stationary points of $\left\langle H_{\mathrm{JJ}}^{\prime}\right\rangle$ in the variational state (3) gives equations for $\lambda$ and $\theta_{i}=\arctan w_{i}$ :

$$
\begin{aligned}
(\omega-\mu)|\lambda| & =\sum_{i} g_{i}^{\prime} \sin \left(2 \theta_{i}\right), \\
\sin \left(2 \theta_{i}\right)\left(\left(\mu^{\prime}-\nu_{i}^{\prime}\right)+2 K_{i}^{\prime} S \cos \left(2 \theta_{i}\right)\right) & =-2 g_{i}^{\prime}|\lambda| \cos \left(2 \theta_{i}\right) .
\end{aligned}
$$

Here primes denote scaled variables, $\mu^{\prime}=\mu S$ etc., and the minimum energy solution has $\sin \left(2 \theta_{i}\right)>0$. For some $N_{\text {ex }}$ and $K_{i}$, the charging energy term in (12) will be negligible. Eqs. 11] and 12 are then just the gap equation (4) derived for polariton condensation, with the replacements $\frac{1}{2} g \rightarrow g^{\prime}$ and $\frac{1}{2}\left(E_{i}-\mu\right) \rightarrow\left(\nu^{\prime}-\mu^{\prime}\right)$.

We have not investigated the consequences of the factors of $S$ and the charging energy in (11) 12). Nevertheless, it seems that the mean-field theories for phaselocking in a Josephson array and for polariton condensation are very similar. This is perhaps surprising, because we tend to think of Bose condensation as a truly quantum phenomenon occurring for microscopic oscillators, while Josephson junctions are macroscopic $(S \gg 1)$, so that phase-locking is naturally thought of in terms of classical coupled oscillators. However as we stressed in the last section, at zero temperature quantum mechanics is irrelevant to the mean-field theory of polariton condensation: the form of the gap equation (4) is the same for quantum spins and for classical angular momenta. The reason for this can be seen in the self-consistent dynamical approach, in which the problem is reduced to that of spins in a self-consistent field. For such a linear problem, the commutativity or otherwise of the spin components is irrelevant. Notice however that there are at least two routes to the classical limit: in the Josephson array, the individual elements become classical as $S \rightarrow \infty$, whereas for the polariton condensate we have $S=1 / 2$, but $N \rightarrow \infty$. In the latter system, only the coherent ground state can be treated as a classical object.

\section{DECOHERENCE OF THE POLARITON CONDENSATE}

Some of the conspicuous differences between quantum and classical oscillators are due to the decoherence of quantum oscillators by their environment. Unlike a classical oscillator, a quantum oscillator has states which do not have a well-defined phase. Furthermore, we expect that the environment will drive it towards such states. Thus we might expect that interactions with the environment would have a significant effect on the phase-locking of quantum oscillators. In fact, we shall see that an infinite condensate is immune to weak decoherence phenomena, in the same way that a superconductor is immune to weak phase-breaking. But in the case of strong decoherence, and perhaps in the case of a finite system, we will find a connection to yet another example of macroscopic phase-locking - the laser.

The laser and the polariton condensate are usually studied in separate contexts, and the connection between them is not made. This should be surprising, as both can be described by exactly the same Hamiltonian (1). However, in a conventional laser 17] the only significant ordering is the coherence of the photons, while in the polariton condensate both the photons and the excitons are 
coherent. This is because in a conventional laser the polarisation of the gain medium, $\left\langle S^{+}\right\rangle$, is strongly damped by processes such as pumping, collisions, and interactions with phonons and impurities. The coherence in the photons remains, because it can be generated by stimulated emmission even from an incoherent reservoir.

The effects on the polariton condensate of different kinds of decoherence processes have recently been studied by two of us [18, 19, 20], using models related to (1). These models are obtained by rewriting each spin operator in terms of a pair of fermions, with annihilation operators $a_{i}$ and $b_{i}$. This is done by replacing $S_{i}^{+}$with $b_{i}^{\dagger} a_{i}$ and $S_{i}^{z}$ with $\frac{1}{2}\left(b_{i}^{\dagger} b_{i}-a_{i}^{\dagger} a_{i}\right)$. With the local constraints $b_{i}^{\dagger} b_{i}+a_{i}^{\dagger} a_{i}=1$ this would give an exact representation of the model (11). In our studies of decoherence, however, we replace these local constraints with their global equivalent. The decoherence is modelled using baths of harmonic oscillators. Thus we consider the Hamiltonian

$$
H=H_{S}+H_{S B}+H_{B} .
$$

The first term $H_{S}$ is just the Hamiltonian (11) written in terms of the fermionic operators,

$H_{S}=\omega_{c} \psi^{\dagger} \psi+\sum_{i=1}^{N} \frac{E_{i}}{2}\left(b_{i}^{\dagger} b_{i}-a_{i}^{\dagger} a_{i}\right)+\frac{g_{i}}{\sqrt{N}}\left(b_{i}^{\dagger} a_{i} \psi+\psi^{\dagger} a_{i}^{\dagger} b_{i}\right)$

$H_{B}$ is a quadratic Hamiltonian describing the baths, and $H_{S B}$ describes the coupling between the system and the baths. The most general form of $H_{S B}$ is

$$
\begin{aligned}
H_{S B} & =\sum_{k} g_{k}^{\kappa}\left(\psi^{\dagger} d_{k}+d_{k}^{\dagger} \psi\right) \\
& +\sum_{i, k}\left[g_{i, k}^{\gamma_{\uparrow}}\left(b_{i}^{\dagger} a_{i} c_{k}^{\alpha \dagger}+c_{k}^{\alpha} a_{i}^{\dagger} b_{i}\right)\right. \\
& +g_{i, k}^{\gamma_{\downarrow}}\left(b_{i}^{\dagger} a_{i} c_{k}^{\beta}+c_{k}^{\beta \dagger} a_{i}^{\dagger} b_{i}\right) \\
& +\Gamma_{k}^{(1)}\left(b_{i}^{\dagger} b_{i}+a_{i}^{\dagger} a_{i}\right)\left(c_{k}^{\zeta \dagger}+c_{k}^{\zeta}\right) \\
& \left.+\Gamma_{i, k}^{(2)}\left(b_{i}^{\dagger} b_{i}-a_{i}^{\dagger} a_{i}\right)\left(c_{k}^{\theta \dagger}+c_{k}^{\theta}\right)\right]
\end{aligned}
$$

The first term in (15) describes the decay of the cavity mode, the second term pumping of the two-level oscillators, while the third term contains all the processes which destroy the electronic excitations, such as sponteneous emmission into modes other than the cavity mode. These baths could give rise to a flow of excitation through the system. The fourth and the fifth terms, however, describe all the dephasing processes which do not change the total number of excitations in the cavity, for example collisions and interactions with phonons and impurities. Processes described by the second, the third and the fourth terms in (15) have pair-breaking character, analogous to magnetic impurities in superconductors, and correspond to potentials which vary rapidly in space or in time.

In order to establish a crossover between an isolated condensate and a laser the decoherence processes must be included self-consistently. The widely used quantum
Maxwell-Bloch (Langevin) equations with a constant decay rate for the polarisation are not correct when the coherent polarisation is large, i.e. for the polariton condensate. In these equations the collective behaviour of the excitons is not taken into account when the lifetime for polarisation is derived. Instead, the lifetime for a single exciton is used in the equation for a collective polarisation mode.

To treat decoherence processes self-consistently, we use a procedure analogous to the Abrikosov-Gor'kov theory [21] of magnetic superconductors. In this theory, the baths which model decoherence are integrated out, introducing effective interactions between different twolevel systems. These interactions are expressed as a selfenergy in Dyson's equation, $G_{i j}^{-1}=G_{0, i j}^{-1}-\Sigma_{i j}$, of the form $\Sigma_{i j}=\gamma G_{i j}$. This form should be contrasted with the non self-consistent treatment, in which the decoherence appears as a constant lifetime in the Dyson equation.

It turns out that the phase-locked polariton condensate is a robust phenomenon because at low decoherence strength it is protected by an energy gap proportional to the photon field amplitude. This gap becomes smaller and finally gets suppressed as the pair-breaking decoherence is increased. At low excitation densities this leads to the suppression of all the coherent fields while at high densities it leads instead to the conventional characteristic of a semiconductor "laser" in which the coherence is almost entirely in the photon field and there is no gap in the excitation spectrum. The laser regime of a polariton system emerges in a way that demonstrates its close analogy to a gapless superconductor.

Although the coherent polarisation in a conventional laser is strongly damped, it must be finite for the laser to operate. Thus the transition between a condensate and a laser is smooth. There is no formal distinction between the two based on the broken symmetry of the ground state. There may be useful practical distinctions however, such as the presence or absence of a gap in the excitation spectrum. We do not yet know if the dynamics of the order parameter, and hence the linewidth, differs. Note that in the Abrikosov-Gor'kov theory the pair-breaking does not produce fluctuations in the order parameter. The theory presented here may share this feature, whereas real lasers have a finite linewidth.

\section{FINITE-SIZE FLUCTUATIONS}

The question of classical or quantum behaviour can never arise for the dynamics of the order parameter in an (infinite) system with a broken symmetry. One simply has a macroscopic equation of motion for the order parameter interacting with an external classical field with a familiar example being the Josephson equation for a weak link, and a less familiar one the classical dynamics of the (averaged) mean field equations in section II. However, when such a system becomes finite (though still large) in extent, we can ask whether the dynam- 
ics are now best descibed by a Schrodinger equation or a Langevin equation: the order parameter will "diffuse", at short times following quantum mechanics, and at longer times dictated by Brownian motion. Of course, we are now concerned only with the low energy degrees of freedom - those near the frequency $\mu$ in the rotating frame - and certainly well away from the quasiparticle excitations above the gap.

The procedure to be followed is clear, at least in principle, though it has not been fully completed for the model of a polariton condensate. As we mentioned briefly above, the variational equations correspond to the saddle point of a quantum mechanical action, which is exact as $N \rightarrow \infty$. Fluctuations at finite $N$ are described by a new effective action, with degrees of freedom that are then coupled to baths exactly as in Eq 15 However, in contrast to the results of the last section, phase-breaking perturbations are expected to be always relevant however weak. We note in passing that this methodology is different from the conventional procedure to begin with a classical action that is then re-quantised. Whether or not it yields any distinct difference is not known.

For a finite system, the broken symmetry ground state will not be stable, and we will observe fluctuations; this is a familiar point of view in classical laser theory, where Haken has emphasised how the mean field theory corresponds to a second-order phase transition, and the fluctuations in a real system arise because the number of photons is not infinite. However, with the starting point of an effective quantum mechanical action one no longer presupposes a classical limit. With the new action one can compute, for example, correlation functions of the photon field, which are directly measurable. At the mean field level, the photon field is a classical electromagnetic field, so the distinction between quantum and classical statistics will only appear at this stage.

\section{DISCUSSION}

The distinctions between coupled oscillators, BEC of polaritons, and strongly damped lasers is an important one in the context of recent experiments on semiconductor microcavities. We will not give a detailed review of the field here, except to point out that one recent experiment 22] has shown evidence for coherence of the photon field in the nonlinear but incoherently pumped microcavity. However, if we define BEC of polaritons to be restricted to systems where the excitonic degrees of freedom have strong coherence, observation of photon coherence is by itself not decisive evidence of polariton BEC. One characteristic of this regime would be a gap in the excitation spectrum, which was not apparently observed.

The difficulties of making these distinctions had already arisen in the Josephson junction array problem here embedded in microwave cavities. As we discussed in section III the Hamiltonian is mathematically sim- ilar to that of the polariton condensate. Recently [23], Barbara et al. reported thresholds in the ac output power of such an array as the driving power was increased. They interpreted their results as analogous to lasing, with gain due to stimulated Josephson tunnelling. Subsequently, Stroud et al. showed that many of the experimental observations could be reproduced by classical treatments [24, 25, 26, 27] of models similar to (9).

The phenomena of lasing and BEC are well-known to be closely connected. At a microscopic level, both are consequences of the quantum mechanics of indistinguishable bosons, and more specifically of stimulated scattering. Lasing is described dynamically, and the role of stimulated scattering is explicit. Bose condensation is described thermodynamically, with the role of stimulated scattering hidden in the Bose-Einstein distribution. On a more sophisticated level, Haken 28] has shown that the mean-field theory of the laser is analogous to that of a second-order phase transition, while Oraevskii 29] has discussed the dynamics of a superconductor in terms of stimulated scattering.

What confuses the issue about BEC is that the conventional textbook picture presents BEC as a consequence of statistical physics of weakly or non- interacting bosons, which obscures the central point that BEC in a macroscopic system is a phase transition like any other. So in a very large system one will not expect to find quantum mechanics operating at the level of the macroscopic order parameter, even if the microscopic theory of this phase transition requires quantum physics. In the JJ array, it is usual to imagine that the individual elements are macroscopic ("decohered") from their environment, but one can see from the above that is is not necessary to assume this in order to develop the correct classical theory of the phase locked coupled array. For our simple model of polariton BEC one can see again that in the large system limit with macroscopic occupancy, the quantum mechanical ground state corresponds to the dynamics of classical phase-locked oscillators. But here there is a possibility to decohere the individual elements (spin half dipoles) from each other (by coupling to external baths) and restore conventional laser theory with a coherent photon field, supported by incoherent electronic polarisation. But still, in the limit of macroscopic occupation, the coherent photon field is essentially a classical one.

Most interesting would be the behaviour of large, but not infinite, systems. Here the order parameter fluctuates generically due to finite size effects. These fluctuations can arise due to environmental interactions, which will give rise to classical diffusion, as in the standard theory of the laser linewidth near threshold. They might also arise because the order parameter tunnels between equivalent states, as has been achieved in systems of small Josephson junctions [30, 31]. The competition between quantum mechanical tunneling and environmental dephasing is of course at the heart of current attempts to create quantum coherent devices - and if excitonic or polaritonic 
BEC were observed, this would provide another possible fundamental system upon which to base such work.

\section{ACKNOWLEDGEMENTS}

PRE and MHS acknowledge the support of research fellowships from Sidney Sussex and Gonville and Caius colleges, Cambridge, respectively. This work is also supported by the EPSRC and by the EU network "Photonmediated phenomena in semiconductor nanostructures".
[1] A. Pikovsky, M. Rosenblum, and J. Kurths, Synchronization (Cambridge University Press, 2001).

[2] A. K. Jain, K. K. Likharev, J. E. Lukens, and J. E. Sauvageau, Phys. Rep. 109, 309 (1984).

[3] M. Borenstein and W. E. Lamb, Jr., Phys. Rev. A 5, 1298 (1972).

[4] C. Weisbuch, M. Nishioka, A.Ishikawa, and Y.Arakawa, Phys. Rev. Lett. 69, 3314 (1992).

[5] M. S. Skolnick, T. A. Fisher, and D. M. Whittaker, Semicond. Sci. Technol. 13, 645 (1998).

[6] J. J. Hopfield, Phys. Rev. 112, 1555 (1958).

[7] L. V. Keldysh, in Bose-Einstein Condensation, edited by A. Griffin, D. W. Snoke, and A. Stringari (Cambridge University Press, Cambridge, U.K., 1995).

[8] S. A. Moskalenko and D. W. Snoke, Bose-Einstein Condensation of Excitons and Biexcitons (CUP, Cambridge, U.K., 2000).

[9] P. R. Eastham and P. B. Littlewood, Sol. Stat. Comm. 116, 357 (2000).

[10] P. R. Eastham, Ph.D. thesis, Cambridge University (2000).

[11] P. R. Eastham and P. B. Littlewood, Phys. Rev. B 64, 235101 (2001).

[12] R. H. Dicke, Phys. Rev. 93, 99 (1954).

[13] D. R. Tilley, Phys. Lett. 33A, 205 (1970).

[14] A. J. Leggett, Rev. Mod. Phys. 73, 307 (2001).

[15] R. Bonificio, F. Casagrande, and M. Milani, Lett. al Nuovo Cimento 34, 520 (1982).

[16] P. A. Lee and M. O. Scully, Phys. Rev. B 3, 769 (1971).

[17] H. Haken, Laser Theory (Springer-Verlag, 1984).

[18] M. H. Szymanska and P. B. Littlewood, Sol. Stat. Comm.
124, 103 (2002).

[19] M. H. Szymanska, Ph.D. thesis, Cambridge University (2001), cond-mat/0204294.

[20] M. H. Szymanska, P. B. Littlewood, and B. D. Simons, cond-mat/0303392.

[21] A. A. Abrikosov and L. P. Gor'kov, JETP 12, 1243 (1961).

[22] H. Deng, G. Weihs, C. Santori, J. Bloch, and Y. Yamamoto, Science 298, 199 (2002).

[23] P. Barbara, A. B. Cawthorne, S. V. Shitov, and C. J. Lobb, Phys. Rev. Lett. 82, 1963 (1999).

[24] J. K. Harbaugh and D. Stroud, Phys. Rev. B 61, 14765 (2000).

[25] E. Almaas and D. Stroud, Phys. Rev. B 67, 064511 (2003).

[26] E. Almaas and D. Stroud, Phys. Rev. B 63, 144522 (2001).

[27] E. Almaas and D. Stroud, Phys. Rev. B 65, 134502 (2002).

[28] H. Haken, Rev. Mod. Phys. 47, 67 (1975).

[29] A. N. Oraevskii, Sov. Phys. JETP 62, 349 (1985).

[30] C. H. van der Wal, A. C. J. ter Haar, F. K. Wilhelm, R. N. Schouten, C. J. P. M. Harmans, T. P. Orlando, S. Lloyd, and J. E. Mooij, Science 290, 773 (2000).

[31] J. R. Friedman, V. Patel, W. Chen, S. K. Tolpygo, and J. E. Lukens, Nature 406, 43 (2000).

[32] Note that the normalisation used for the spin operators in Refs. 15 and 16 differs from that required by our definitions. For example, $S^{z}$ in Ref. 16 is the difference in density, rather than number, of pairs. 